\title{
Closed-form transformation between geodetic and ellipsoidal coordinates
}

W.E. Featherstone (corresponding author)

Western Australian Centre for Geodesy \& The Institute for Geoscience Research, Curtin University of Technology, GPO Box U1987, Perth, WA 6845, Australia;

Phone: +61 89266 2734; Fax: +61 89266 2703; Email: W.Featherstone@curtin.edu.au

\section{S.J. Claessens}

Western Australian Centre for Geodesy \& The Institute for Geoscience Research, Curtin University of Technology, GPO Box U1987, Perth, WA 6845, Australia;

Phone: +61 89266 3505; Fax: +61892662703; Email: S.Claessens@curtin.edu.au

\begin{abstract}
We present formulas for direct closed-form transformation between geodetic coordinates $(\phi, \lambda, h)$ and ellipsoidal coordinates $(\beta, \lambda, u)$ for any oblate ellipsoid of revolution. These will be useful for those dealing with ellipsoidal representations of the Earth's gravity field or other oblate ellipsoidal figures. The numerical stability of the transformations for nearpolar and near-equatorial regions is also considered.
\end{abstract}

Keywords: Geodetic coordinates, ellipsoidal coordinates, coordinate transformations, ellipsoidal geodesy

\section{Generic notation}

a semi-major axis length of an ellipsoid

$b=a(1-f) \quad$ semi-minor axis length of an ellipsoid

$f=(a-b) / a \quad$ flattening of an ellipsoid [dimensionless]

$e=\sqrt{2 f-f^{2}}$ first numerical eccentricity of an ellipsoid [dimensionless]

$E=a e \quad$ linear eccentricity (or focal length) of an ellipsoid

$(\phi, \lambda, h) \quad$ geodetic coordinates: latitude, longitude and height

$(\beta, \lambda, u) \quad$ ellipsoidal coordinates: ellipsoidal co-latitude, longitude and ellipsoidal parameter

$(X, Y, Z) \quad$ 3D Cartesian coordinates aligned with the ellipsoids' axes 


\section{Introduction}

\subsection{Geodetic coordinates $(\phi, \lambda, h)$}

Transformation between geodetic coordinates (geodetic latitude $\phi$, longitude $\lambda$ and ellipsoidal/geodetic height $h)$ and 3D Cartesian coordinates $(X, Y, Z)$ has been studied repeatedly over the years (Table 1). It has attracted the attention of not only geodesists, but also aerospace engineers and others. Curiously, there seems to have been little cross-fertilization of ideas, with some being repeated in the literature.

\section{Table 1 near here}

It is first instructive to point out that while several of the studies cited in Table 1 term this $(\phi, \lambda, h) \leftrightarrow(X, Y, Z)$ coordinate transformation 'geocentric from/to Cartesian', it need not be. It is equally applicable to non-geocentric geodetic ellipsoids, such as those used in conjunction with local geodetic datums, which is needed for Helmert-type horizontal datum transformations. Likewise, the geodetic from/to ellipsoidal coordinate $(\beta, \lambda, u)$ transformations presented here do not have to be geocentric.

\section{Figure 1 near here}

Referring to Fig. 1, the origin of the Cartesian coordinate system must coincide exactly with the geometrical centre (not the focii) of the geodetic ellipsoid used as the reference surface for the geodetic $\phi, \lambda, h$ coordinate system. The Cartesian $Z$ axis must lie along the minor axis of the geodetic ellipsoid, pointing positively towards the north pole. Not shown in Fig. 1, the Cartesian $X$ axis must lie in the equatorial plane of the geodetic ellipsoid, pointing positively towards the Greenwich meridian, by convention. The Cartesian $Y$ axis completes a righthanded coordinate system, thus pointing positively towards $90^{\circ} \mathrm{E}$ in the equatorial plane. In Fig. $1, W^{2}=X^{2}+Y^{2}$.

The geodetic ellipsoid is the geometrical figure mapped by an oblate (i.e., flattened towards the poles) ellipse rotated about its minor axis. It is sometimes synonymously called a spheroid in the geodetic literature (e.g.,Bomford 1980). The geodetic coordinate system is two-parametric, requiring the a priori selection of the numerical values of the semi-major axis $a_{g}$ and first numerical eccentricity $e_{g}$ (or the latter's equivalents: flattening $f_{g}$ or semi-minor axis $b_{g}$ ). The subscript $g$ is used to distinguish the parameters for the geodetic ellipsoid, so as to maintain generality throughout.

Again referring to Fig. 1, the geodetic latitude $(-\pi / 2 \leq \phi \leq \pi / 2)$ is the angle between the 
equatorial plane ( $X-Y$ plane or $W$ axis in Fig. 1) and the normal to the surface of the geodetic ellipsoid that passes through the point of interest $\mathrm{P}$, measured positively northwards in the meridional $(Z-W)$ plane. The ellipsodial/geodetic height $h$ is the distance along the surface normal from the geodetic ellipsoid to the point of interest $\mathrm{P}$, measured positively outwards. Not shown in Fig. 1, the geodetic longitude $\lambda(-\pi \leq \lambda \leq \pi)$ is the angle between the Greenwich meridian ( $Z-X$ plane) and the meridian through the point of interest $\mathrm{P}$ ( $Z-W$ plane), often measured positively eastwards and negatively westwards in the equatorial plane.

Under these conditions, the geodetic coordinates $(\phi, \lambda, h)$ are related to the corresponding Cartesian coordinates $(X, Y, Z)$ by (e.g.,Heiskanen and Moritz 1967, Bowring 1976, Pollard 2002); cf. Fig. 1:

$$
\begin{aligned}
& X=\left(\nu_{g}+h\right) \cos \phi \cos \lambda \\
& Y=\left(\nu_{g}+h\right) \cos \phi \sin \lambda \\
& Z=\left\{\nu_{g}\left(1-e_{g}^{2}\right)+h\right\} \sin \phi
\end{aligned}
$$

where the radius of curvature in the prime vertical of the surface of the geodetic ellipsoid is (e.g.,Bomford 1980; Vaníček and Kleusberg 1982)

$$
\nu_{g}=\frac{a_{g}}{\sqrt{1-e_{g}^{2} \sin ^{2} \phi}}=\frac{a_{g}^{2}}{\sqrt{a_{g}^{2} \cos ^{2} \phi+b_{g}^{2} \sin ^{2} \phi}}
$$

This forward transformation $(\phi, \lambda, h \rightarrow X, Y, Z)$ is both straightforward and numerically stable, but the reverse $(X, Y, Z \rightarrow \phi, \lambda, h)$ is not so. This has led to a plethora of iterative, approximative and closed-form transformation equations have been presented (Table 1). However, the user must be careful when interpreting some authors' claims of a closed-form or non-iterative equation, because most of them really only hold this property for $h=0$.

Numerical comparisons among some of the methods in Table 1 have been conducted by, e.g., Laskowski (1991), Barrio and Riaguas (1993), Gerdan and Deakin (1999), Seemkooei (2002), Fok and Bâki Iz (2003) and Burtch (2006), in addition to comparisons made by the proposers of some of the approaches cited in Table 1. As far as we are aware, no comprehensive comparison of all the different methods has yet been made, but that is beyond the scope of this paper. However, the 2003 IERS Standards (McCarthy and Petit 2004; http://www.iers.org/documents/publications/tn endorse Borkowski's (1989) method. 


\subsection{Ellipsoidal coordinates $(\beta, \lambda, u)$}

A relatively less-well-known coordinate system, but which is well-suited to describing positions on, in and above the Earth's surface, is the [oblate] ellipsoidal coordinate system, comprising the ellipsoidal co-latitude $\beta$, ellipsoidal longitude $\lambda$ and ellipsoidal parameter $u$ (Fig. 1). This is a special degenerate case of the spheroidal [i.e., triaxial ellipsoid] coordinate system (e.g.,Hobson 1931) that is of much more geodetic relevance.

One notable benefit is that the ellipsoidal coordinate system is triply orthogonal and thus permits a separation of variables for the solution of Laplace's equation, resulting in ellipsoidal harmonics (e.g. Darwin 1901a,b, Hobson 1931, Byerly 1959, Heiskanen and Moritz 1967, Moon and Spencer 1988). As such, it is particularly well-suited to representing the Earth's gravity field (e.g. Jekeli 1988, Thông and Grafarend 1989, Sansò and Sona 2001, Finn and Grafarend 2005, Bölling and Grafarend 2005, Grafarend et al. 2006).

While a complete separation of variables can be achieved in ellipsoidal, spherical-polar or Cartesian coordinates, it cannot be achieved for the geodetic coordinates, apart from on the surface (i.e., $h=0$ ) of the geodetic ellipsoid (Grafarend 1988). This inseparability problem arises because $h$ and $\phi$ are closely related in the geodetic coordinate system (see Eqs. 1 to 4). However, this has not prevented geodetic coordinates from uniquely locating points on or near the Earth's surface, as hundreds of years of geodetic practice do testify.

Due to this inseparability, full 3D harmonic analysis of the Earth's gravity field is not possible in geodetic coordinates. Instead, spherical or ellipsoidal harmonics have to be used, with spherical harmonics still being the most popular for gravity field synthesis (e.g. see the list of spherical harmonic Earth gravity models at the International Centre for Global Earth Models (ICGEM), http://icgem.gfz-potsdam.de/ICGEM/ICGEM.html).

Also in this case, the origin of the Cartesian coordinate system must also coincide exactly with the geometrical centre (not the focii) of the confocal ellipsoids comprising the ellipsoidal coordinate system; the Cartesian $X$ axis lies in the equatorial plane towards the Greenwich meridian; the Cartesian $Z$ axis lies along the minor axis of the confocal ellipsoids forming the $u$ coordinate in the ellipsoidal coordinate system; the Cartesian $Y$ axis completes a righthanded rectangular system.

Therefore, the Cartesian origin and axes are perfectly aligned for both the geodetic and ellipsoidal coordinate systems. If not, they can easily be aligned using a six-parameter Helmert transformation. There is no need to employ a scale change when dealing with a rigid coordinate 
system; see Featherstone and Vaníček (1999) or Vaníček et al. (2002).

The ellipsoidal co-latitude ( $0 \leq \beta \leq \pi$ ) is the angle, measured positively southwards in the meridional $(Z-W)$ plane, between the minor axis of the ellipsoidal coordinates (or the Cartesian $Z$ axis) and the radius vector from the origin of the confocal ellipsoids to a point on a circumscribing sphere (radius $\sqrt{u^{2}+E^{2}}$ ) above the point of interest $\mathrm{P}$ on a line parallel to the minor axis of the confocal ellipsoids (Fig. 1). The ellipsoidal parameter $u$ defines the length of the semi minor axis of each confocal ellipsoid. Coupled with the linear eccentricity parameter $E$, this causes a unique confocal ellipsoid ( $u=$ constant) to pass through the point of interest. The ellipsoidal longitude $\lambda$ is identical to the geodetic longitude when the corresponding Cartesian axes are aligned.

Under these conditions, the ellipsoidal coordinates $(\beta, \lambda, u)$ are related to Cartesian coordinates $(X, Y, Z)$ by (e.g. Hobson 1931, Heiskanen and Moritz 1967, Eq. 1-103; Vaníček and Krakiwsky 1982; Gleason 1988), cf. Fig. 1:

$$
\begin{aligned}
& X=\sqrt{u^{2}+E_{e}^{2}} \sin \beta \cos \lambda \\
& Y=\sqrt{u^{2}+E_{e}^{2}} \sin \beta \sin \lambda \\
& Z=u \cos \beta
\end{aligned}
$$

where, in this deliberately generic case, the linear eccentricity is

$$
E_{e}^{2}=a_{e}^{2} e_{e}^{2}=a_{e}^{2}-b_{e}^{2}
$$

where the subscript $e$ is used to distinguish the ellipsoidal coordinate system. Finally, the ellipsoidal coordinate system is one-parametric and triply orthogonal.

Throughout this paper, only the geodetically relevant case of oblate ellipsoidal coordinates will be used. The special degenerate case when $E_{e}$ and $u$ together yield the form of the geodetic ellipsoid will be given later. Prolate ellipsoids are not considered because they are of lesser geodetic relevance, but the equations here can be adapted to that case if needed.

In this paper, we will present direct closed-form forward and reverse transformations between geodetic and ellipsoidal coordinates. As well as being of curiosity-driven interest (we are currently unaware of any previously presented work explicitly on this, apart from a passing mention in Vaníček and Krakiswky (1982)), this transformation will be of use, e.g., to those 
who want to directly display the results from an ellipsoidal harmonic synthesis on a map, among other applications.

The motivation is to give a one-stage closed-form transformation between 3D geodetic and 3D ellipsoidal coordinates, thus eliminating the arbitrary error introduced by using iterative techniques. We acknowledge that this transformation could also be achieved by staging it via Cartesian coordinates (e.g., geodetic to Cartesian then Cartesian to ellipsoidal and vice versa), but a one-stage transformation is attractive for reasons of simplicity, and it will run faster if coded well (cf.Fukushima 2006).

\section{Geodetic to ellipsoidal transformation: $(\phi, \lambda, h) \rightarrow(\beta, \lambda, u)$}

By definition, the longitudes in the geodetic and ellipsoidal coordinate systems are identical when the corresponding Cartesian axes are aligned, which is evident by inspection of Eqs. (1) and (2) and Eqs. (5) and (6). Dividing Eqs. (5) and (6) yields

$$
\lambda=\arctan \frac{Y}{X}
$$

where the signs of $X$ and $Y$ are used to select the appropriate quadrant for the longitude $\lambda$. However, there are some limitations on the numerical stability of Eq. (9). Vaníček and Krakiswky (1982 p.) give a form that is numerically more stable near the poles, which is

$$
\lambda=2 \arctan \frac{Y}{X+\sqrt{X^{2}+Y^{2}}}
$$

Vermeille (2004) points out that Eq. (10) is still problematic, giving a more numerically stable variant

$$
\begin{array}{ll}
\lambda=\frac{\pi}{2}-2 \arctan \frac{X}{\sqrt{X^{2}+Y^{2}}+Y} & \text { for } Y \geq 0 \\
\lambda=-\frac{\pi}{2}+2 \arctan \frac{X}{\sqrt{X^{2}+Y^{2}}-Y} & \text { for } Y<0
\end{array}
$$

However, this coordinate will not be considered further here because the focus of this paper is not on this transformation as the geodetic and ellipsoidal longitudes are identical.

The geodetic to ellipsoidal coordinate transformation thus reduces to $(\phi, h) \rightarrow(\beta, u)$. First, we consider the most general case for an arbitrary geodetic ellipsoid $\left(a_{g}, e_{g}\right)$ and an arbitrary [oblate] ellipsoidal coordinate system. 
In order to find the ellipsoidal parameter $u$ from the geodetic $\phi$ and $h$, we first sum the squares of Eqs. (1) and (2) and equate this to the sum of the squares of Eqs. (5) and (6)

$$
\left(\nu_{g}+h\right)^{2} \cos ^{2} \phi=\left(u^{2}+E_{e}^{2}\right) \sin ^{2} \beta
$$

Rearranging gives

$$
\sin ^{2} \beta=\frac{\left(\nu_{g}+h\right)^{2} \cos ^{2} \phi}{\left(u^{2}+E_{e}^{2}\right)}
$$

Next, we equate the squares of Eqs. (7) and (3)

$$
\left(\nu_{g}\left(1-e_{g}^{2}\right)+h\right)^{2} \sin ^{2} \phi=u^{2} \cos ^{2} \beta
$$

and rearranging gives

$$
\cos ^{2} \beta=\frac{\left(\nu_{g}\left(1-e_{g}^{2}\right)+h\right)^{2} \sin ^{2} \phi}{u^{2}}
$$

Eliminating the ellipsoidal co-latitude $\beta$ by taking the sum of Eqs. (14) and (16) gives

$$
\frac{\left(\nu_{g}+h\right)^{2} \cos ^{2} \phi}{\left(u^{2}+E_{e}^{2}\right)}+\frac{\left(\nu_{g}\left(1-e_{g}^{2}\right)+h\right)^{2} \sin ^{2} \phi}{u^{2}}=1
$$

which reduces to a biquadratic equation in $u$ (quadratic equation in $u^{2}$ ), according to

$$
\begin{gathered}
u^{4}+u^{2}\left[E_{e}^{2}-\left(\nu_{g}+h\right)^{2} \cos ^{2} \phi-\left(\nu_{g}\left(1-e_{g}^{2}\right)+h\right)^{2} \sin ^{2} \phi\right] \ldots \\
-E_{e}^{2}\left(\nu_{g}\left(1-e_{g}^{2}\right)+h\right)^{2} \sin ^{2} \phi=0
\end{gathered}
$$

which has the solution

$$
\begin{gathered}
u=\left(\frac{1}{2}\left[\left(\nu_{g}+h\right)^{2} \cos ^{2} \phi+\left(\nu_{g}\left(1-e_{g}^{2}\right)+h\right)^{2} \sin ^{2} \phi-E_{e}^{2}\right]\right. \\
\left.\ldots+\sqrt{\frac{1}{4}\left[E_{e}^{2}-\left(\nu_{g}+h\right)^{2} \cos ^{2} \phi-\left(\nu_{g}\left(1-e_{g}^{2}\right)+h\right)^{2} \sin ^{2} \phi\right]^{2}+E_{e}^{2}\left(\nu_{g}\left(1-e_{g}^{2}\right)+h\right)^{2} \sin ^{2} \phi}\right)^{\frac{1}{2}}
\end{gathered}
$$

where the positive square root has been taken for the second term on the right-hand side of Eq. (19) in order to have a real square root for the whole of the right-hand side.

Once the numerical value of the geodetic parameter $u$ has been found from Eq. (19), the ellipsoidal co-latitude $\beta$ is given by either the square root of Eq. (14)

$$
\sin \beta=\frac{\left(\nu_{g}+h\right) \cos \phi}{\sqrt{u^{2}+E_{e}^{2}}}
$$

or the square root of Eq. (16)

$$
\cos \beta=\frac{\left(\nu_{g}\left(1-e_{g}^{2}\right)+h\right) \sin \phi}{u}
$$


one of which is selected depending on the proximity to the poles or equator so as to avoid roundoff errors in their computation (through the sine and cosine terms). An alternative formula for non-polar regions is given by division

$$
\tan \beta=\frac{u}{\sqrt{u^{2}+E_{e}^{2}}} \frac{\left(\nu_{g}+h\right)}{\left(\nu_{g}\left(1-e_{g}^{2}\right)+h\right)} \cot \phi
$$

Finally, we consider the degenerate case when the geodetic parameter $u$ and linear eccentricity $E_{e}$ in the oblate ellipsoidal coordinate system are chosen to coincide exactly with the geodetic ellipsoid, or vice versa. Accordingly, $h$ must equal zero in the geodetic coordinate system. We also note that for any ellipsoid

$$
a^{2}=E^{2}+u^{2}
$$

Therefore, since $u=b_{g}=b_{e}$, then $a_{g}^{2}=a_{e}^{2}=\left(E_{g}^{2}+b_{g}^{2}\right)=\left(E_{e}^{2}+b_{e}^{2}\right)$. Inserting these degenerating conditions in Eq. (22) gives, on the surface of the geodetic ellipsoid,

$$
\tan \beta=\frac{a_{g}}{b_{g}} \cot \phi=\frac{a_{e}}{b_{e}} \cot \phi
$$

which is a variant of the well-known relation between the geodetic and reduced latitudes for points on the geodetic ellipsoid (e.g. Bomford 1980, Eq. A.52). The difference here is because we are dealing with the reduced co-latitude $\beta$ (cf. Fig. 1).

\section{Ellipsoidal to geodetic transformation: $(\beta, \lambda, u) \rightarrow(\phi, \lambda, h)$}

As stated in the Introduction, one option to achieve this transformation is to stage the computations via Cartesian coordinates, i.e., $(\beta, \lambda, u) \rightarrow(X, Y, Z) \rightarrow(\phi, \lambda, h)$. However, the latter transformation is cumbersome, needing accuracy criteria if one selects an approximative or iterative solution (cf. Table 1).

Therefore, we seek a direct closed-form solution for $(\beta, \lambda, u) \rightarrow(\phi, \lambda, h)$, actually $(\beta, u)$ $\rightarrow(\phi, h)$, that does not require these intermediate transformation stages. This offers savings in computational cost (cf. Fukushima 2006) and is not subject to considerations of accuracy versus number of iterations (cf. Keeler and Nievergelt 1998).

\subsection{Special case for $u=b_{g}$ (equivalently $h=0$ )}

First, we show the special case for points already known to be located on the surface of the geodetic ellipsoid, i.e. $h=0$ in the geodetic coordinate system. Thus, this transformation 
simplifies to $(\beta, u) \rightarrow(\phi)$. Dividing the square root of Eq. (13) by the square root of Eq. (15), noting that $h=0$, gives

$$
\tan \phi=\frac{u}{\left(1-e_{g}^{2}\right) \sqrt{u^{2}+E_{e}^{2}}} \cot \beta=\frac{u a_{g}^{2}}{b_{g}^{2} \sqrt{u^{2}+E_{e}^{2}}} \cot \beta
$$

A further specific degeneration can be made when the ellipsoidal coordinate system is chosen such that it coincides with the surface of the geodetic ellipsoid, or vice versa, to give

$$
\tan \phi=\frac{a_{g}}{b_{g}} \cot \beta=\frac{a_{e}}{b_{e}} \cot \beta
$$

Equations (25) and (26) are closed-form because of the a priori condition of $h=0$. This is expected because closed-form equations exist for the transformation from Cartesian to geodetic coordinates when $h=0$ (cf. Bowring 1976). It also a by-product of the ability to separate geodetic coordinates for Laplace's equation on the surface of the geodetic ellipsoid (cf. Grafarend 1988).

\subsection{General case for $u \neq b_{g}$ (equivalently all $h$ )}

Equating Eqs. (1) and (5) or, identically, equating Eqs.(2) and (6), gives

$$
\left(\nu_{g}+h\right) \cos \phi=\sqrt{u^{2}+E_{e}^{2}} \sin \beta
$$

then rearranging gives

$$
h=\frac{\sqrt{u^{2}+E_{e}^{2}} \sin \beta}{\cos \phi}-\nu_{g}
$$

Likewise, equating Eqs. (3) and (7) gives

$$
\left(\nu_{g}\left(1-e_{g}^{2}\right)+h\right) \sin \phi=u \cos \beta
$$

and rearranging gives

$$
h=\frac{u \cos \beta}{\sin \phi}-\nu_{g}\left(1-e_{g}^{2}\right)
$$

Equations (28) and (30) require the geodetic latitude $\phi$ be known, which is our next aim. Dividing them leaves (cf. Eq. 22)

$$
\tan \phi=\left(\frac{u}{\sqrt{u^{2}+E_{e}^{2}}}\right)\left(\frac{\left(\nu_{g}+h\right)}{\left(\nu_{g}\left(1-e_{g}^{2}\right)+h\right)}\right) \cot \beta
$$

which is an iterative formula because $\nu_{g}$ is a function of $\phi$. A starting value for the iteration is given by Eq. (25), but the rate of convergence will depend on the magnitude of the ellipsoidal height $h$. Bowring's (1976) formula could also be used as a starting value. However, 
the problem with iterative solutions is that users have to run numerical tests to determine the desired convergence criterion, which is of concern for accuracy-critical applications (cf. Keeler and Nievergelt 1998), and approximative solutions are naturally subject to the approximation errors.

Instead, here we prefer a closed-form solution. Essentially this is a minimum-distance mapping (Helmert projection; cf. Grafarend and Lohse 1991; Crocetto 1993) problem for a point with respect to the geodetic ellipsoid, which can be solved using quartic equations (e.g. Paul 1973, Vaníček and Krakiwsky 1982, Borkowski 1987, 1989; Ozone 1995), Lagrange parameters (e.g. Zhang et al. 2005) or vector methods (e.g. Feltens 2007).

\subsubsection{Paul's (1973) closed-form solution}

Here, we choose Paul's (1973) quartic solution for the sake of clarity. From a few computational tests, we found virtually no difference in computation speed between this and Borkowski's (1989) method. The computational speed could be accelerated by coding to avoid divisions or the use of transcendental functions as much as possible (cf. Fukushima 2006), but we coded Paul's (1973) algorithm as published and used Borkowski's (1989) original code, which is replicated in the 2003 IERS Standards (McCarthy and Petit 2004;

http://www.iers.org/documents/publications/tn/tn32/tn32.pdf). We leave such optimisation to those who are interested, noting that low-cost computer speed increases almost as fast as the time that additional coding and testing for the optimisation would take.

Eliminating $h$ from Eqs. (28) and (30) gives

$$
\frac{\sqrt{u^{2}+E_{e}^{2}} \sin \beta}{\cos \phi}=\frac{u \cos \beta}{\sin \phi}+e_{e}^{2} \nu_{g}
$$

Inserting the second form of Eq. (4) in Eq. (32) and simplifying yields

$$
\frac{a_{g}^{2} e_{g}^{2} \sin \phi}{\sqrt{a_{g}^{2} \cos ^{2} \phi+b_{g}^{2} \sin ^{2} \phi}}=\sqrt{u^{2}+E_{e}^{2}} \sin \beta \tan \phi-u \cos \beta
$$

Dividing both numerator and denominator of the left-hand side by $\cos \phi$, then squaring both sides and simplifying gives

$$
a_{g}^{4} e_{g}^{4} \tan ^{2} \phi=\left(a_{g}^{2}+b_{g}^{2} \tan ^{2} \phi\right)\left(\sqrt{u^{2}+E_{e}^{2}} \sin \beta \tan \phi-u \cos \beta\right)^{2}
$$

then multiplying out and collecting like terms in $\tan \phi$ gives the quartic equation

$$
\left(b_{g}^{2}\left(u^{2}+E_{e}^{2}\right) \sin ^{2} \beta\right) \tan ^{4} \phi-\left(2 b_{g}^{2} \sqrt{u^{2}+E_{e}^{2}} \sin \beta u \cos \beta\right) \tan ^{3} \phi
$$




$$
\begin{aligned}
& \ldots+\left(a_{g}^{2}\left(u^{2}+E_{e}^{2}\right) \sin ^{2} \beta+u^{2} b_{g}^{2} \cos ^{2} \beta-a_{g}^{4} e_{g}^{4}\right) \tan ^{2} \phi \\
& \ldots-\left(2 a_{g}^{2} \sqrt{u^{2}+E_{e}^{2}} \sin \beta u \cos \beta\right) \tan \phi-a_{g}^{2} u^{2} \cos ^{2} \beta=0
\end{aligned}
$$

A quartic equation can be solved using Ferrari's method (e.g. Weisstein 1999, pp.14891491), which achieves its solution by eliminating the cubic term. This approach was used by Borkowski (1989), through a particular substitution, but requires some selection of the appropriate root. Instead, here we apply the result from Paul (1973), which relies on the solution of a quartic equation through elimination of the quadratic term. This approach was applied to Eq. (35) to give a solution for $\tan \phi$

$$
\tan \phi=\frac{\left(\frac{u \cos \beta}{2}+\sqrt{D}+\sqrt{\frac{u^{2} \cos ^{2} \beta}{4}-\frac{B}{2}-D+\frac{a_{g} u \cos \beta}{4 \sqrt{D}}}\right)}{\sqrt{u^{2}+E_{e}^{2}} \sin \beta}
$$

where

$$
\begin{aligned}
& A=\frac{\left(u^{2}+E_{e}^{2}\right) \sin ^{2} \beta+a_{g}^{2} e_{g}^{4}}{1-e_{g}^{2}} \\
& B=\frac{\left(u^{2}+E_{e}^{2}\right) \sin ^{2} \beta-a_{g}^{2} e_{g}^{4}}{1-e_{g}^{2}} \\
& C=1+\frac{27 u^{2} \cos ^{2} \beta\left(A^{2}-B^{2}\right)}{2\left(u^{2} \cos ^{2} \beta+B\right)^{3}} \\
& D=\frac{\left(u^{2} \cos ^{2} \beta+B\right)}{12}\left[\left(C+\sqrt{C^{2}-1}\right)^{1 / 3}+\left(C+\sqrt{C^{2}-1}\right)^{-1 / 3}\right] \\
& \ldots-\frac{B}{6}+\frac{u^{2} \cos ^{2} \beta}{12}
\end{aligned}
$$

Both Borkowski's (1989) and Paul's (1973) methods become numerically unstable near the equator. Therefore, an additional IF STATEMENT is needed. Our few numerical tests also showed that there was no significant timing difference when this was added (cf. Fukushima 2006). As such, Paul's (1973, Eq. 9') should be used close (say $<5^{\circ}$ ) to the equator.

\subsubsection{Vermeille's (2002; 2004) closed-form solution}

However, Vermeille $(2002 ; 2004)$ has presented closed form equations, also based on the solution of a quartic equation, for the $(\phi, \lambda, h) \leftrightarrow(X, Y, Z)$ transformation that are numerically stable near the equator and poles. Therefore, users may prefer these algorithms so as to avoid the use of IF STATEMENTS in Borkowski's (1989) and Paul's (1973) solutions. 
Adapting the algorithm given in Vermeille (2002; Section 3) and Vermeille (2004; Section 2) to the notation used in this paper gives

$$
\phi=2 \arctan \frac{u \cos \beta}{F+\sqrt{F^{2}+u^{2} \cos ^{2} \beta}}
$$

where

$$
\begin{aligned}
& F=\frac{G \sqrt{u^{2}+E_{e}^{2}} \sin \beta}{G+e_{g}^{2}} \\
& G=\sqrt{H+I+J^{2}}-J \\
& H=K\left(1+L+L^{-1}\right) \\
& I=\sqrt{H^{2}+e_{g}^{4} M} \\
& J=\frac{e_{g}^{2}}{2 I}(H+I-M) \\
& K=\frac{M+N-e_{g}^{4}}{6} \\
& L=\sqrt[3]{1+O+\sqrt{2 O+O^{2}}} \\
& M=\frac{\left(1-e_{g}^{2}\right) u^{2} \cos ^{2} \beta}{a_{g}^{2}} \\
& N=\frac{\left(u^{2}+E_{e}^{2}\right) \sin ^{2} \beta}{a_{g}^{2}} \\
& O=e_{g}^{4} M N \\
& 4 K^{3}
\end{aligned}
$$

Since Eqs. (41) to (51) contain fewer transcendental functions than Eqs. (36) to (40), Vermeille's (2002) algorithm, if coded properly, will also run faster than Paul's (1973); cf. Fukushima (2006).

\section{Concluding remarks}

We have presented closed-form equations (plus one iterative) to transform between geodetic coordinates $(\phi, \lambda, h)$ and ellipsoidal coordinates $(\beta, \lambda, u)$. These will be of use, e.g., for those dealing with ellipsoidal harmonic expressions of the Earth's external gravity field, among other applications. The benefit of closed-form equations is that the numerical errors are controllable 
(e.g., by careful computer coding), rather than having to run tedious numerical experiments for all likely (and perhaps unlikely) scenarios (cf. Keeler and Nievergelt 1998). Toms, RM (SRI International, personal communication, 2006) advised us that a new ISO/IEC 18026 Spatial Reference Model standard on this issue was released as an International Standard in 2006 (http://standards.sedris.org/18026/index.htm). This may be where closed-form equations, such as ours, may be beneficial.

Acknowledgements: Will Featherstone gratefully acknowledges the generous support of the Alexander von Humboldt Stiftung through award number 1120942, as well as the generous hospitality of all staff in the Geodätisches Institut, Universität Stuttgart, Germany. Vielen Danke! Will Featherstone and Sten Claessens also gratefully acknowledge funding from the Australian Research Council (ARC) through grant DP0663020. We also thank Ralph Toms for providing information on the ISO Standard as part of our ongoing correspondence. Finally, thanks are extended to the editor and three reviewers of this manuscript. This is The Institute for Geoscience Research publication number 83.

\section{References}

Barbee TW (1982) Geodetic latitude of a point in space, Journal of Spacecraft and Rockets 19(4):378-380

Barrio R, Riaguas A (1993) Comparison of algorithms for the transformation from geocentric to geodetic coordinates, Rev Acad Cienc Zaragoza 48(2):135-143

Bartelme N, Meissl P (1975) Ein einfaches, rasches und numerisch stabiles Verfahren zur Bestimmung des kürzesten Abstandes eines Punktes von einem sphäroidischen Rotationsellipsoid, Allgemeine VermessungsNachrichten 82(12):436-439

Bencini P (1968) Sul calcolo di transformazione dal sistemia geocentrico al systemia geografico, Bollettino di Geodesia Scienze Affini 27(3):357-367

Benning W (1974) Der kürzeste Abstand eines in rechtwinkligen Koordinaten gegebenen Außenpunktes vom Ellipsoid, Allgemeine Vermessungs-Nachrichten 81(11):429-433

Benning W (1987) Iterative ellipsoidische Lofußpunktberechnung, Allgemeine Vermessungs-Nachrichten 94(7):256-260

Berger WJ, Ricupito JR (1960) Geodetic latitude and altitude of a satellite, Journal of Advanced Robotic Systems 30(9):901-902

Bölling K, Grafarend EW (2005) Ellipsoidal spectral properties of the Earth's gravitational potential and its first and second derivatives, Journal of Geodesy 79(6-7):300-330, doi: 10.1007/s00190-005-0465-y Bomford G (1980) Geodesy, 4th Edition, Oxford University Press, Oxford

Bopp H, Krauss H (1976) Der Kürzeste Abstand eines Punktes von einem Rotationsellipsoid, Allgemeine 
Vermessung-Nachrichten 83(10):348-351

Borkowski KM (1987) Transformation of geocentric to geodetic coordinates without approximations, Astrophysics and Space Science 139:1-4 [erratum in 146(1):201]

Borkowski KM (1989) Accurate algorithms to transform geocentric to geodetic coordinates, Bulletin Géodésique 63(1):50-56

Bowring BR (1976) Transformation from spatial to geographical coordinates, Survey Review 23(181):323327

Bowring BR (1985) The accuracy of geodetic latitude and height equations, Survey Review 28(218):202206

Burtch R (2006) A comparison of methods used in rectangular to geodetic coordinate transformations, Proceedings of the American Congress on Surveying and Mapping (ACSM) Annual Conference and Technology Exhibition, Orlando, April 21-26, http://www.acsm.net/sessions06/Burtch424.pdf

Byerly WE (1959) An Elementary Treatise on Fourier's Series, and Spherical, Cylindrical, and Ellipsoidal Harmonics, with Applications to Problems in Mathematical Physics, Dover, New York

Carlson NA (1980) Fast geodetic coordinate transformations, Proceedings of Guidance and Control Conference, Danvers, August 11-13, pp 328-337

Churchyard JN (1986) Comment on 'A new algorithm for the computation of the geodetic coordinates as a function of earth-centered earth-fixed coordinates', Journal of Guidance, Control, and Dynamics 9(4):511-511

Crocetto N (1993) Point projection of the topographic surface onto the reference ellipsoid of revolution in geocentric Cartesian coordinates, Survey Review 32(250):233-238

Darwin GH (1901a) Ellipsoidal harmonic analysis, Philosophical Transactions of the Royal Society of London: Series A, Containing Papers of a Mathematical or Physical Character 197:461-557

Darwin GH (1901b) Ellipsoidal harmonic analysis, Proceedings of the Royal Society of London 68:248252

Deprit A, Deprit-Bartholome A (1975) Conversion from geocentric to geodetic coordinates, Celestial Mechanics 12:489-493

Ecker E (1967) Die Normaenfällung auf das Ellipsoid, Österrich Zeitschrift für Vermessung und Photogrammetrie 3(1):82-85

Featherstone WE, Vaníček (1999) The role of coordinate systems, coordinates and heights in horizontal datum transformations, The Australian Surveyor 44(2):143-150 [Correspondence in 45(1):5-11 and The Trans-Tasman Surveyor 1(3):5-7]

Feltens J (2007) Vector methods to compute azimuth, elevation, ellipsoidal normal, and the Cartesian $(\mathrm{X}, \mathrm{Y}, \mathrm{Z})$ to geodetic $(\phi, \lambda, \mathrm{h})$ transformation, J Geod (in press)

Finn G, Grafarend EW (2005) Ellipsoidal vertical deflections: regional, continental, global maps of the horizontal derivative of the incremental gravity potential, in Sansò F (ed.) V Hotine-Marussi Symposium on Mathematical Geodesy, Springer, Berlin Heidelberg New York, pp 252-259 
Fok HS, Bâki Iz H (2003) A comparative analysis of the performance of iterative and non-iterative solutions to the Cartesian to geodetic coordinate transformation, Journal of Geospatial Engineering 5(2):6174

Fotiou A (1998) A pair of closed expressions to transform geocentric to geodetic coordinates, Zeitschrift für Vermessungswesen 123(4):133-135

Frölich H, Hansen HH (1976) Zur Lotfußpunktberechnung bei rotationsellipsoidischer Bezugsfläche, Allgemeine Vermessungs-Nachrichten 83(5):175-179

Fukushima T (1999) Fast transform from geocentric to geodetic coordinates, Journal of Geodesy 73(11):603610, doi: 10.1007/s001900050271

Fukushima T (2006) Transformation from Cartesian to geodetic coordinates accelerated by Halley's method, Journal of Geodesy 79(12):689-693, doi: 10.1007/s00190-006-0023-2

Gerdan GP, Deakin RE (1999) Transforming Cartesian coordinates $X, Y, X$ to geographical coordinates $\phi, \lambda, h$, The Australian Surveyor 44(1):55-63

Gersten RH (1961) Geodetic sub-latitude and altitude of a space vehicle, Journal of Astronautical Sciences 8(1):28-29

Gleason DM (1988) Comparing ellipsoidal corrections to the transformation between the geopotential's spherical and ellipsoidal spectrums, manuscripta geodaetica 13(2):114-129

Grafarend EW (1988) The geometry of the Earth's surface and the corresponding function space of terrestrial gravitational field. in: Festschrift to R Sigl, Rep B287, German Geodetic Commission, Munich, pp. 76-94

Grafarend EW (2001) Gauss surface normal coordinates in geometry and gravity space part 2a: topographic surface normal ellipsoidal coordinates: closed form transformation of "geocentric Cartesian" to "Gauss ellipsoidal coordinates (geodetic)", Zeitschrift für Vermessungswesen 126(6):373-382

Grafarend EW, Lohse P (1991) The minimal distance mapping of the topographic surface onto the (reference) ellipsoid of revolution, manuscripta geodaetica 16(1):92-110

Grafarend EW, Finn G, Ardalan AA (2006) Ellipsoidal vertical deflections and ellipsoidal gravity disturbance: case studies. Studia Geophysica et Geodaetica 50(1):1-57, doi: 10.1007/s11200-006-0001-4

Guo G (2001) A fast procedure for geodetic coordinate transformation, Surveying and Mapping 11(1):1213

Heck B (1987) Rechenverfahren und Auswertemodelle der Landesvermessung, Wichmann, Karlsruhe Hedgely DR (1976) An exact transformation from geocentric to geodetic coordinates for non-zero altitudes, Technical Report R-458, NASA, Maryland

Hedman EL (1970) A high-accuracy relationship between geocentric Cartesian coordinates and geodetic latitude and altitude, Journal of Spacecraft and Rockets 7(8):993-995

Heikkinen M (1982) Geschlossene Formeln zur Berechnung räumlicher geodätischer Koordinaten aus rechtwinkligen Koordinaten, Zeitschrift für Vermessungswesen 107(5):207-211 
Heindl G (1997) How to compute interval inclusions of geodetic coordinates from interval inclusions of Cartesian coordinates, Reliable Computing 3(4):421-435, doi: 10.1023/A:1009953605448

Heiskanen WA, Moritz H (1967) Physical Geodesy, Freeman, San Francisco

Hekimoğlu S (1995) Generalized iterative solution for geodetic coordinates from Cartesian coordinates, Bollettino Geodesia e Scienze Affini 54(1):109-120

Hirvonen RA (1964) Praktische Rechenformeln für die dreidimensionale Geodäsie, Zeitschrift für Vermessungswesen 89(5):145-151

Hirvonen R, Moritz H (1963) Practical computation of gravity at high altitudes, Report 27, Institute of Geodesy, Photogrammetry and Cartography, Ohio State University, Columbus

Hobson EW (1931) The Theory of Spherical and Ellipsoidal Harmonics, Cambridge University Press, New York

Hsu DY (1992) Closed form solution for geodetic coordinates transformations. Proceedings of the National Technical Meeting of The Institute of Navigation, San Diego, January, pp. 397-400

Jekeli C (1988) The exact transformation between ellipsoidal and spherical harmonic expansions, manuscripta geodaetica 13(2):106-113

Jones GC (2002) New solutions for the geodetic coordinate transformation, Journal of Geodesy 76(8):437446, doi: 10.1007/s00190-002-0267-4

Keeler SP, Nievergelt Y (1998) Computing geodetic coordinates, SIAM Review 40(2):300-309

Lapaine M (1990) A new direct solution of the transformation problem of Cartesian into ellipsoidal coordinates, in: Rapp RH, Sansò (eds) Determination of the Geoid: Present and Future, Springer, Berlin Heidelberg New York, pp 395-404

Laskowski P (1991) Is Newton's iteration faster than simple iteration for transformation between geocentric and geodetic coordinates? Bulletin Géodésique 65(1):14-17, doi: 10.1007/BF00806337

Lester Jones E.(1922) Conversion of rectangular to geodetic co-ordinates, The Geographical Journal 60(1):90

Levin JZ (1988) A rational parametric approach to latitude, longitude, and altitude, Navigation 35(3):361370

Lin K-C, Wang J (1995) Transformation from geocentric to geodetic coordinates using Newton's iteration, Bulletin Géodésique 69(4):300-303, doi 10.1007/BF008067

Long SAT (1975) General-altitude transformations between geocentric and geodetic coordinates, Celestial Mechanics 12:225-230

Lupash LO (1985) A new algorithm for the computation of the geodetic coordinates as a function of Earth-centered Earth-fixed coordinates. Journal of Guidance, Control, and Dynamics 8(6):787-789

Mayer M (1978) Theoretische Untersuchen über die Genauigkeit der dreidimensionalen terrestrischen Punktbestimmung, German Geodetic Commission, Reihe C, Hefte 244, Munich

McCarthy DD, Petit G (Eds.) (2004) International Earth Rotation and Reference Systems Service (IERS) Conventions (2003), IERS Technical Note 32, Verlag des Bundesamts fr̈ Kartographie und Geodäsie, 
Frankfurt am Main

Morrison J, Pines S (1961) The reduction from geocentric to geodetic coordinates, Astronomical Journal $66(1): 15-16$

Moon P, Spencer DE (1988) Field Theory Handbook, Including Coordinate Systems, Differential Equations, and Their Solutions, 2nd ed., Springer, Berlin Heidelberg New York

Nautiyal A (1988) Algorithm to generate geodetic coordinates from Earth-centered Earth-fixed coordinates, Journal of Guidance, Control, and Dynamics 11(3):281-283

Nievergelt Y, Keeler SP (2000) Computing geodetic coordinates in space, Journal of Spacecraft and Rockets 37(2):293-296

Olson DK (1988) Calculation of geodetic coordinates from Earth-centered Earth-fixed coordinates, Journal of Guidance Control and Dynamics 11(2):188-190

Olson DK (1996) Converting Earth-centered, Earth-fixed coordinates to geodetic coordinates, IEEE Transactions on Aerospace and Electronic Systems 32(1):473-476, doi: 10.1109/7.481290

Ozone MI (1985) Non-iterative solution of the $\phi$ equation, Surveying and Mapping 45(2):169-171

Paul MK (1973) A note on computation of geodetic coordinates from geocentric (Cartesian) coordinates, Bulletin Géodésique 108(2):135-139

Pavlov K (1967) Sur la transformation de coordonnees rectangulaires speciales $X, Y, Z$ en coordonnees geographiques $\phi, \lambda, h$ pour les hauteurs inferieures a $10 \mathrm{~km}$, Schweizerische Zeitschrift für Vermessung, Photogrammetrie und Kulturtechnik 6(12):381-388

Penev P (1978) The transformation of rectangular coordinates into geographical by closed formulas, Geodesy Mapping and Photogrammetry 20:175-177

Pick M (1967) Transformation of the spatial rectangular coordinates into the geodetic coordinates, Bulletin Géodésique 83(1):21-26

Pick M (1985) Closed formulas for the transformation of the Cartesian coordinate system into a system of geodetic coordinates, Studia Geophysica et Geodetica 29:112-119

Pollard J (2002) Iterative vector methods for computing geodetic latitude and height from rectangular coordinates. Journal of Geodesy 76(1):36-40, doi: 10.1007/s001900100220

Pollard J (2005) A new approach to the iterative calculation of geodetic latitude and its calculation, Survey Review 38(296):117-123

Purcell EW, Cowan WB (1961) Relating geodetic latitude and altitude to geocentric latitude and radius vector, Journal of Advanced Robotic Systems 31(7):932-934

Rinner K (1958) Geometrie mit Raumstrecken, Zeitschrift für Vermessungswesen 89(3):91-105

Sansò F, Sona G (2001) ELGRAM: an ellipsoidal gravity model manipulator, Bollettino di Geodesia e Scienze Affini 60(3):215-226

Seemkooei AA (2002) Comparison of different algorithms to transform geocentric to geodetic coordinates, Survey Review 36(286):627-633 
Sjöberg LE (1999) An efficient iterative solution to transform rectangular geocentric coordinates to geodetic coordinates, Zeitschrift für Vermessungswesen 124(3):295-297

Sofair I (1997) Improved method for calculating exact geodetic latitude and altitude, Journal of Guidance, Control, and Dynamics 20(4):824-826

Sofair I (2000) Improved method for calculating exact geodetic latitude and altitude revisited, Journal of Guidance, Control, and Dynamics 23(2):369

Soler T, Hothem LD (1989) Important parameters used in geodetic transformations, Journal of Surveying Engineering 115(4):414-417

Sudano JJ (1997) An exact conversion from an Earth-centered coordinate system to latitude, longitude and altitude, Proceedings of the IEEE National Aerospace and Electronics Conference 1997 (NAECON 1997), vol. 2, Dayton, 14-17 July, pp 646-650

Sugai I (1967) Note on exact geodetic sub-latitude and geodetic altitude of a space vehicle, Journal of the Astronautical Sciences 14(3):134-137

Sünkel H (1999) Ein nicht-iteratives Verfahren zur Transformation geodätischer Koordinaten, Österlich Zeitschrift für Vermessungswesen 64:29-33

Thông NC, Grafarend EW (1989) A spheroidal harmonic model of the terrestrial gravitational field, manuscripta geodaetica 14(5):285-304

Toms RM (1995) An efficient algorithm for geocentric to geodetic coordinate conversion, Proceedings on Standards for the Interoperability of Distributed Simulations, Volume 1, Institute for Simulation and Training, Orlando, September, pp 635-642

Toms RM (1996) An improved algorithm for geocentric to geodetic coordinate conversion, Proceedings on Standards for the Interoperability of Distributed Simulations, Volume 2, Institute for Simulation and Training, Orlando, February, pp 1135-1144

Toms RM (1998) New efficient procedures for geodetic coordinate transformations, Proceedings on Standards for the Interoperability of Distributed Simulations, Volume 3, Institute, for Simulation and Training, Orlando, March, pp 1024-1033

Vaníček P, Krakiwsky (1982) Geodesy: The Concepts, Elsevier, Amsterdam

Vaníček P, Novák P, Craymer M, Pagiatakis SD (2002) On the correct determination of transformation parameters of a horizontal geodetic datum, Geomatica 56(4):329-340

Vermeille H (2002) Direct transformation from geocentric to geodetic coordinates, Journal of Geodesy 76(8):451-454, doi: 10.1007/s00190-002-0273-6

Vermeille H (2004) Computing geodetic coordinates from geocentric coordinates, Journal of Geodesy 78(1-2):94-95, doi: 10.1007/s00190-004-0375-4

Vincenty T (1976) Ein Verfahren zur Bestimmung der geodaetischen Hoehe eines Punktes, Allgemeine Vermessungs-Nachrichten 83(5):179

Vincenty T (1978) Vergleich zweier Verfahren zur Berechnung der geodätischen Breite und Höhe aus rechwinkligen Koordinaten, Allgemeine Vermessungs-Nachrichten 85(7):269-270 
Vincenty T (1980) Zur räumlich-ellipsoidischen Koordinaten-Transformation, Zeitschrift für Vermessungswesen 105(11):519-521

Weisstein EW (1999) CRC Concise Encyclopedia of Mathematics, CRC Press, Boca Raton, 1969pp

Wu Y, Wang P, Hu X (2003) Algorithm of Earth-centered Earth-fixed coordinates to geodetic coordinates, IEEE Transactions on Aerospace and Electronic Systems 39(45):1457-1461

You R-J (2000) Transformation of Cartesian to geodetic coordinates without iterations, Journal of Surveying Engineering 126(1):1-7, doi:10.1061/(ASCE)0733-9453(2000)126:1(1)

Zhang CD, Hsu HT, Wu XP, Li SS, Wang QB, Chai HZ, Du L (2005) An alternative algebraic algorithm to transform Cartesian to geodetic coordinates, Journal of Geodesy 79(8):413-420, doi: 10.1007/s00190005-0487-5

Zhu J (1993) Exact conversion of Earth-centered, Earth-fixed coordinates to geodetic coordinates. Journal of Guidance Control and Dynamics 16(2):389-391

Zhu J (1994) Conversion of Earth-centered Earth-fixed coordinates to geodetic coordinates. IEEE Transactions on Aerospace and Electronic Systems 30(3):957-961 


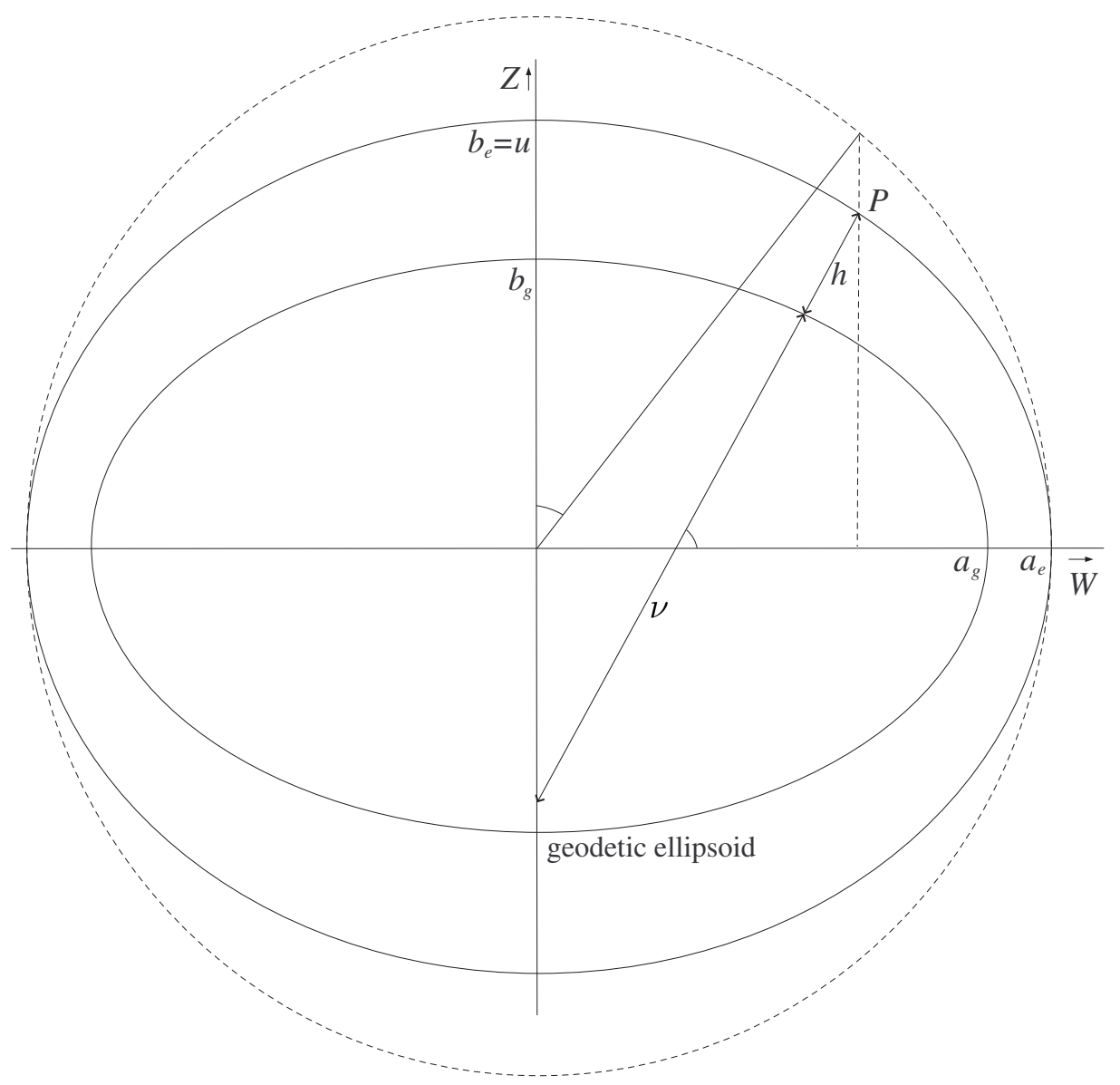

Figure 1: Meridional cross section (because of rotational symmetry) of the relationships among 2D Cartesian $(Z, W)$, geodetic $(\phi, h)$ and [oblate] ellipsoidal $(\beta, u)$ coordinates for an arbitrary point $\mathrm{P}$. 
Table 1: A list of studies on the $(X, Y, Z) \leftrightarrow(\phi, \lambda, h)$ coordinate transformation, with closed-form solutions identified by CF. Studies published in the Russian and Eastern European literature are not cited; instead see Lapaine (1990)

\begin{tabular}{|l|l|}
\hline Barbee (1982) & Bartelme and Meissl (1975) \\
\hline Bencini (1968) & Benning (1974, 1987) \\
\hline Berger and Ricupito (1960) & Bopp and Krauss (1976) \\
\hline Borkowsky (1987, 1989) CF & Bowring (1976, 1985) \\
\hline Carlson (1980) & Churchyard (1986) \\
\hline Crocetto (1993) & Deprit and Deprit-Bartholome (1975) \\
\hline Ecker (1967) CF & Fotiou (1998) CF \\
\hline Frölich and Hansen (1976) CF & Fukushima (1999, 2006) \\
\hline Gen (1981) & Gersten (1961) \\
\hline Grafarend (2001) CF & Grafarend and Lohse (1991) \\
\hline Guo (2001) & Heck (1987) \\
\hline Hedgely (1976) CF & Hedman (1970) \\
\hline Heikkinen (1982) CF & Heindl (1997) \\
\hline Heiskanen and Moritz (1967) & Hekimoğlu (1995) \\
\hline Hirvonen (1964) & Hirvonen and Moritz (1963) \\
\hline Hsu (1992) CF & Jones (2002) CF \\
\hline Keeler and Nievergelt (1998) & Lapaine (1990) CF \\
\hline Laskowski (1991) & Lester Jones (1922) \\
\hline Levin (1988) & Lin and Wang (1995) \\
\hline Long (1975) & Lupash (1985) \\
\hline Mayer (1978) & Morrison and Pines (1961) \\
\hline Nautiyal (1988) & Nievergelt and Keeler (2000) \\
\hline Olson (1988) & Ozone (1985) CF \\
\hline Paul (1973) CF & Pavlov (1967) \\
\hline Penev (1978) CF & Pick (1967, 1985) CF \\
\hline Pollard (2002, 2005) & Purcell and Cowan (1961) \\
\hline Rinner (1978) & Sjöberg (1999) \\
\hline Sofair (1997, 2000) & Soler and Hothem (1989) \\
\hline Sugai (1967) CF & Sünkel (1999) CF \\
\hline Toms (1995, 1996, 1998) & Vermeille (2002, 2004) CF \\
\hline Vincenty (1976, 1978, 1980) & Wu et al. (2003) \\
\hline You (2000) & Zhang et al. (2005) CF \\
\hline Zhu (1993, 1994) & Feltens (2007) \\
\hline Sudano (1997) & Olson (1996) \\
\hline
\end{tabular}

